\title{
DIRECTLY DECOMPOSABLE IDEALS AND CONGRUENCE KERNELS OF COMMUTATIVE SEMIRINGS
}

\author{
IVAN CHAJDA, GÜNTHER EIGENTHALER, AND HELMUT LÄNGER
}

Received 18 January, 2019

\begin{abstract}
As pointed out in the monographs [5,6] on semirings, ideals play an important role despite the fact that they need not be congruence kernels as in the case of rings. Hence, having two commutative semirings $\mathbf{S}_{1}$ and $\mathbf{S}_{2}$, one can ask whether an ideal $I$ of their direct product $\mathbf{S}=\mathbf{S}_{1} \times \mathbf{S}_{2}$ can be expressed in the form $I_{1} \times I_{2}$ where $I_{j}$ is an ideal of $\mathbf{S}_{j}$ for $j=1,2$. Of course, the converse is elementary, namely if $I_{j}$ is an ideal of $\mathbf{S}_{j}$ for $j=1,2$ then $I_{1} \times I_{2}$ is an ideal of $\mathbf{S}_{1} \times \mathbf{S}_{2}$. Having a congruence $\Theta$ on a commutative semiring $\mathbf{S}$, its 0 -class is an ideal of $\mathbf{S}$, but not every ideal is of this form. Hence, the lattice $\mathbf{I d} \mathbf{S}$ of all ideals of $\mathbf{S}$ and the lattice $\operatorname{Ker} \mathbf{S}$ of all congruence kernels (i.e. 0-classes of congruences) of $\mathbf{S}$ need not be equal. Furthermore, we show that the mapping $\Theta \mapsto[0] \Theta$ need not be a homomorphism from $\operatorname{ConS}$ onto KerS. Moreover, the question arises when a congruence kernel of the direct product $\mathbf{S}_{1} \times \mathbf{S}_{2}$ of two commutative semirings can be expressed as a direct product of the corresponding kernels on the factors. In the paper we present necessary and sufficient conditions for such direct decompositions both for ideals and for congruence kernels of commutative semirings. We also provide sufficient conditions for varieties of commutative semirings to have directly decomposable kernels.
\end{abstract}

2010 Mathematics Subject Classification: 16Y60; 08A05; 08B10; 08A30

Keywords: semiring, congruence, ideal, skew ideal, congruence kernel, direct decomposability

\section{INTRODUCTION}

The importance of the investigation of semirings is based on the fact that they form a concept which covers both distributive lattices with 0 and rings. Hence, several results known for rings can be transformed to distributive lattices and vice versa. Of course, the concept of a semiring is much more general than that of a ring and hence not all the results known for rings can be extended to semirings. In particular, rings are congruence permutable contrary to the case of semirings. Further, for rings, congruences are in a one-to-one correspondence with ideals. This does not hold for semirings. However, important results on rings motivate the investigation of similar

Support of the research of the first and third author by the Austrian Science Fund (FWF), project I 4579-N, and the Czech Science Foundation (GAČR), project 20-09869L, as well as by ÖAD, project CZ 02/2019, and, concerning the first author, by IGA, project PřF 2020 014, is gratefully acknowledged. 
results on semirings. In the present paper we study the relation between ideals and congruence kernels with respect to direct decomposability.

There exist two different versions of the concept of a semiring, namely semirings having a unit element ([5]) and those without such an element $([3,6])$. Since the second version is mostly used in applications, we define the basic concept of this paper as follows:

Definition 1 (see [6]). A commutative semiring is an algebra $\mathbf{S}=(S,+, \cdot, 0)$ of type $(2,2,0)$ satisfying

- $(S,+, 0)$ is a commutative monoid,

- $(S, \cdot)$ is a commutative semigroup,

- $(x+y) z \approx x z+y z$

- $0 x \approx 0$.

If $\mathbf{S}$ is a commutative semiring containing an element 1 satisfying the identity $1 x \approx x$ then $\mathbf{S}$ is called unitary, and if it satisfies the identity $x x \approx x$ then it is called idempotent. A (semi-)ring $(S,+, \cdot, 0)$ is called zero-(semi-)ring if $x y=0$ for all $x, y \in$ $S$.

It is evident that every (unitary) commutative ring is a (unitary) commutative semiring and that every distributive lattice $\mathbf{L}=(L, \vee, \wedge, 0)$ with least element 0 is an idempotent commutative semiring.

In the following let $\mathbb{N}$ denote the set of non-negative integers. Then clearly, $\mathbf{N}=$ $(\mathbb{N},+, \cdot, 0)$ is a unitary commutative semiring. For every positive integer $a$ let $a \mathbb{N}$ denote the set $\{0, a, 2 a, 3 a, \ldots\}$ of all non-negative multiples of $a$. It is evident that $a \mathbf{N}=(a \mathbb{N},+, \cdot, 0)$ is again a commutative semiring which is unitary if and only if $a=1$.

We recall the following definition from [5]:

Definition 2. Let $\mathbf{S}=(S,+, \cdot, 0)$ be a commutative semiring. An ideal of $\mathbf{S}$ is a subset $I$ of $S$ satisfying

- $0 \in I$,

- if $a, b \in I$ then $a+b \in I$,

- if $a \in I$ and $s \in S$ then $a s \in I$.

Note that in case that $\mathbf{S}$ is a ring, the ideals of $\mathbf{S}$ in the sense of Definition 2 need not be ring ideals. Consider e.g. the zero-ring whose additive group is the group $(\mathbb{Z},+, 0)$ of the integers. Then the ideals of this zero-ring in the sense of Definition 2 are the submonoids of $(\mathbb{Z},+, 0)$, whereas the ring ideals are the subgroups of $(\mathbb{Z},+, 0)$.

The converse is, of course, trivial for any ring $\mathbf{S}$ : Every ring ideal of $\mathbf{S}$ is an ideal in the sense of Definition 2.

Let Id $\mathbf{S}$ denote the set of all ideals of a commutative semiring $\mathbf{S}=(S,+, \cdot, 0)$. It is clear that $\mathbf{I d} \mathbf{S}=(\operatorname{Id} \mathbf{S}, \subseteq)$ is a complete lattice with smallest element $\{0\}$ and greatest 
element $S$. Moreover, for $I, J \in \operatorname{Id} \mathbf{S}$ we have

$$
\begin{aligned}
& I \vee J=I+J=\{i+j \mid i \in I, j \in J\}, \\
& I \wedge J=I \cap J .
\end{aligned}
$$

For $a \in S$ let $I(a)$ denote the ideal of $\mathbf{S}$ generated by $a$. Obviously, $I(a)=a \mathbb{N}+a S$. The lattice Id $\mathbf{S}$ need not be modular as the following example shows:

Example 1. The Hasse diagram of the ideal lattice of the commutative zero-semiring $\mathbf{S}=(S,+, \cdot, 0)$ on $\{0, a, b, c, d, e, f, g\}$ defined by

\begin{tabular}{c|llllllll}
+ & 0 & $a$ & $b$ & $c$ & $d$ & $e$ & $f$ & $g$ \\
\hline 0 & 0 & $a$ & $b$ & $c$ & $d$ & $e$ & $f$ & $g$ \\
$a$ & $a$ & $b$ & $c$ & 0 & $e$ & $f$ & $g$ & $d$ \\
$b$ & $b$ & $c$ & 0 & $a$ & $f$ & $g$ & $d$ & $e$ \\
$c$ & $c$ & 0 & $a$ & $b$ & $g$ & $d$ & $e$ & $f$ \\
$d$ & $d$ & $e$ & $f$ & $g$ & $d$ & $e$ & $f$ & $g$ \\
$e$ & $e$ & $f$ & $g$ & $d$ & $e$ & $f$ & $g$ & $d$ \\
$f$ & $f$ & $g$ & $d$ & $e$ & $f$ & $g$ & $d$ & $e$ \\
$g$ & $g$ & $d$ & $e$ & $f$ & $g$ & $d$ & $e$ & $f$
\end{tabular}

looks as follows (see Figure 1):

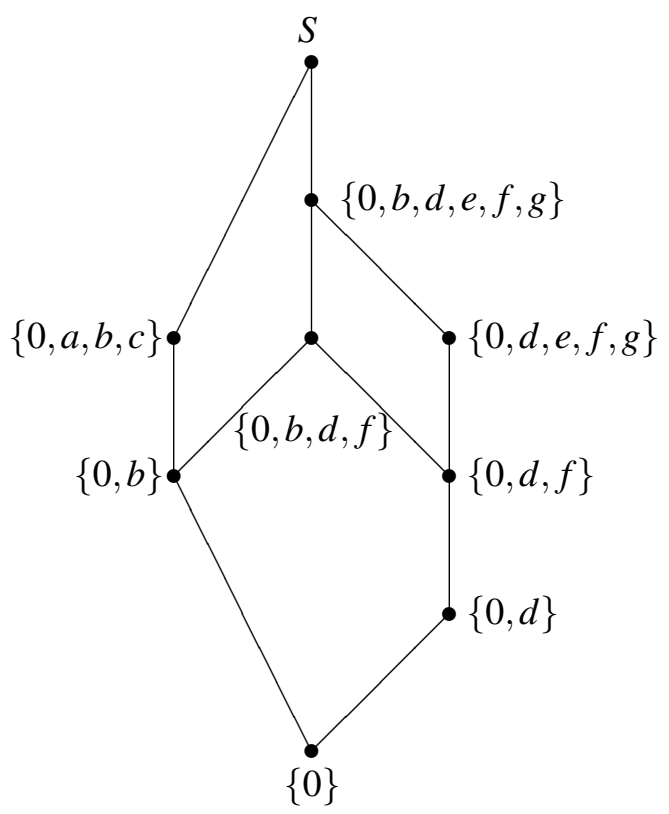

FIGURE 1. 
and hence this lattice is not modular. Observe that $(S,+, 0)$ is isomorphic to the direct product of its submonoids $\{0, a, b, c\}$ (four-element cyclic group) and $\{0, d\}$ (two-element join-semilattice). The ideals of the semiring $\mathbf{S}$ are the submonoids of $(S,+, 0)$.

Let $\operatorname{Con} \mathbf{S}=(\operatorname{Con} \mathbf{S}, \subseteq)$ denote the congruence lattice of a commutative semiring S. A congruence kernel of $\mathbf{S}$ is a set of the form $[0] \Theta$ with $\Theta \in$ Con $\mathbf{S}$. It is well known (cf. [5]) that every congruence kernel is an ideal of $\mathbf{S}$, but not vice versa. Let

$$
\operatorname{Ker} \mathbf{S}=(\operatorname{Ker} \mathbf{S}, \subseteq)=(\{[0] \Theta \mid \Theta \in \operatorname{Con} \mathbf{S}\}, \subseteq)
$$

denote the (complete) lattice of congruence kernels of $\mathbf{S}$. In contrast to rings, $\mathbf{C o n} \mathbf{S}$ and $\operatorname{Ker} \mathbf{S}$ need not be isomorphic as the following example shows, in which two different congruences have the same kernel.

Example 2. Consider the three-element lattice $\mathbf{D}_{3}=\left(D_{3}, \vee, \wedge, 0\right)=$ $(\{0, a, 1\}, \vee, \wedge, 0)$. Then the Hasse diagram of $\mathbf{C o n}_{3}$ looks as follows (see Figure 2):

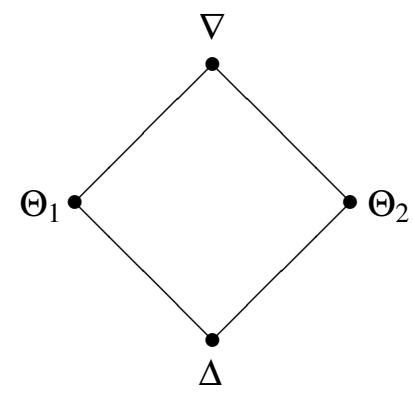

FigURE 2.

where

$$
\Theta_{1}=\{0, a\}^{2} \cup\{1\}^{2} \text { and } \Theta_{2}=\{0\}^{2} \cup\{a, 1\}^{2} .
$$

However, $\Delta$ and $\Theta_{2}$ have the same kernel $\{0\}$. Hence

$$
\operatorname{Ker}_{3}=\left(\left\{\{0\},\{0, a\}, D_{3}\right\}, \subseteq\right)
$$

is a three-element chain and $\operatorname{Con}_{3} \neq \operatorname{Ker} \mathbf{D}_{3}$. Moreover, even the mapping $\Theta \mapsto$ $[0] \Theta$ is not a homomorphism from $\mathbf{C o n}_{\mathbf{3}}$ onto $\operatorname{Ker} \mathbf{D}_{3}$ since

$$
[0]\left(\Theta_{1} \vee \Theta_{2}\right)=[0] \nabla=D_{3} \neq\{0, a\}=\{0, a\} \vee\{0\}=[0] \Theta_{1} \vee[0] \Theta_{2}
$$




\section{IDEALS OF DIRECT PRODUCTS OF COMMUTATIVE SEMIRINGS}

In the following we are interested in ideals on a direct product of two commutative semirings. Let $\mathbf{S}_{1}$ and $\mathbf{S}_{2}$ be commutative semirings. Of course, if $I_{1} \in \operatorname{Id} \mathbf{S}_{1}$ and $I_{2} \in \operatorname{Id} \mathbf{S}_{2}$ then $I_{1} \times I_{2} \in \operatorname{Id}\left(\mathbf{S}_{1} \times \mathbf{S}_{2}\right)$. An ideal $I$ of $\mathbf{S}_{1} \times \mathbf{S}_{2}$ is called directly decomposable if there exist $I_{1} \in \operatorname{Id} \mathbf{S}_{1}$ and $I_{2} \in \operatorname{Id} \mathbf{S}_{2}$ with $I_{1} \times I_{2}=I$. If $I$ is not directly decomposable then it is called a skew ideal. The aim of this paper is to characterize those commutative semirings which have directly decomposable ideals.

Example 3. If $\mathbf{R}_{2}=\left(R_{2},+, \cdot, 0\right)=(\{0,1\},+, \cdot, 0)$ denotes the two-element zeroring and $\mathbf{D}_{2}=\left(D_{2}, \vee, \wedge, 0\right)=(\{0,1\}, \vee, \wedge, 0)$ the two-element lattice then $\mathbf{S}:=\mathbf{R}_{2} \times$ $\mathbf{D}_{2}$ has the non-trivial ideals

$$
\begin{aligned}
& \{0\} \times D_{2}, \\
& R_{2} \times\{0\}, \\
& \{(0,0),(0,1),(1,1)\}
\end{aligned}
$$

and hence $\mathbf{I d} \mathbf{S} \cong \mathbf{N}_{5}$ which is not modular and, moreover, the last mentioned ideal is skew.

Example 4. If $\mathbf{R}_{4}=\left(R_{4},+, \cdot, 0\right)=(\{0, a, b, c\},+, \cdot, 0)$ denotes the zero-ring whose additive group is the Kleinian 4-group defined by

\begin{tabular}{c|cccc}
+ & 0 & $a$ & $b$ & $c$ \\
\hline 0 & 0 & $a$ & $b$ & $c$ \\
$a$ & $a$ & 0 & $c$ & $b$ \\
$b$ & $b$ & $c$ & 0 & $a$ \\
$c$ & $c$ & $b$ & $a$ & 0
\end{tabular}

then $\mathbf{S}:=\mathbf{R}_{4} \times \mathbf{D}_{2}$ has the non-trivial ideals

$$
\begin{aligned}
& \{0, a\} \times\{0\}, \\
& \{0, b\} \times\{0\}, \\
& \{0, c\} \times\{0\}, \\
& R_{4} \times\{0\}, \\
& \{0\} \times D_{2}, \\
& \{0, a\} \times D_{2}, \\
& \{0, b\} \times D_{2}, \\
& \{0, c\} \times D_{2}, \\
& \{(0,0),(0,1),(a, 1)\}, \\
& \{(0,0),(0,1),(b, 1)\}, \\
& \{(0,0),(0,1),(c, 1)\},
\end{aligned}
$$




$$
\begin{aligned}
& \{(0,0),(0,1),(a, 1),(b, 1),(c, 1)\}, \\
& \{(0,0),(a, 0),(0,1),(a, 1),(b, 1),(c, 1)\}, \\
& \{(0,0),(b, 0),(0,1),(a, 1),(b, 1),(c, 1)\}, \\
& \{(0,0),(c, 0),(0,1),(a, 1),(b, 1),(c, 1)\}
\end{aligned}
$$

the last seven of which are skew.

For sets $A$ and $B$ let $\pi_{1}$ and $\pi_{2}$ denote the first and second projection from $A \times B$ onto $A$ and $B$, respectively. Note that for any subset $C$ of $A \times B$ we have $C \subseteq \pi_{1}(C) \times$ $\pi_{2}(C)$. Furthermore, if $C$ is of the form $A_{1} \times B_{1}$ with $A_{1} \subseteq A$ and $B_{1} \subseteq B$ then $\pi_{1}(C)=$ $A_{1}$ and $\pi_{2}(C)=B_{1}$.

We borrow the method from [4] (which was used also in [2]) to prove the following theorem:

Theorem 1. Let $\mathbf{S}_{1}=\left(S_{1},+, \cdot, 0\right)$ and $\mathbf{S}_{2}=\left(S_{2},+, \cdot, 0\right)$ be commutative semirings and $I \in \operatorname{Id}\left(\mathbf{S}_{1} \times \mathbf{S}_{2}\right)$ and consider the following assertions:

(i) I is directly decomposable,

(ii) $\left(S_{1} \times\{0\}\right) \cap\left(\left(\{0\} \times S_{2}\right)+I\right) \subseteq I$ and $\left(\left(S_{1} \times\{0\}\right)+I\right) \cap\left(\{0\} \times S_{2}\right) \subseteq I$,

(iii) if $(a, b) \in I$ then $(a, 0),(0, b) \in I$,

(iv) $\left(\left(S_{1} \times\{0\}\right)+I\right) \cap\left(\left(\{0\} \times S_{2}\right)+I\right)=I$.

Then (iii) $\Leftrightarrow$ (i) $\Rightarrow$ (iv) $\Rightarrow$ (ii).

\section{Proof.}

(iii) $\Rightarrow$ (i): If $(a, b) \in \pi_{1}(I) \times \pi_{2}(I)$ then there exists some pair $(c, d) \in S_{1} \times S_{2}$ with $(a, d),(c, b) \in I$, hence $(a, 0),(0, b) \in I$ which shows $(a, b)=(a, 0)+(0, b) \in I$.

(i) $\Rightarrow$ (iii): This is clear.

(i) $\Rightarrow$ (iv): If $I=I_{1} \times I_{2}$ then

$$
\begin{aligned}
& \left(\left(S_{1} \times\{0\}\right)+I\right) \cap\left(\left(\{0\} \times S_{2}\right)+I\right)= \\
& =\left(\left(S_{1} \times\{0\}\right)+\left(I_{1} \times I_{2}\right)\right) \cap\left(\left(\{0\} \times S_{2}\right)+\left(I_{1} \times I_{2}\right)\right)= \\
& =\left(S_{1} \times I_{2}\right) \cap\left(I_{1} \times S_{2}\right)=I_{1} \times I_{2}=I .
\end{aligned}
$$

(iv) $\Rightarrow$ (ii): This follows immediately.

Remark 1. That (ii) does not imply (iii) can be seen by considering the ideal $I:=$ $\{(0,0),(0,1),(a, 1)\}$ of $\mathbf{S}$ in Example 4. Since

$$
\begin{aligned}
& \left(S_{1} \times\{0\}\right) \cap\left(\left(\{0\} \times S_{2}\right)+I\right)=\{(0,0)\} \subseteq I, \\
& \left(\left(S_{1} \times\{0\}\right)+I\right) \cap\left(\{0\} \times S_{2}\right)=\{(0,0),(0,1)\} \subseteq I,
\end{aligned}
$$

(ii) holds. Because of $(a, 1) \in I$ and $(a, 0) \notin I$, (iii) does not hold. This shows that (ii) does not imply (iii). It is worth noticing that the implication (ii) $\Rightarrow$ (iii) holds in the case of commutative rings since in this case

$$
(a, 0)=(0,-b)+(a, b)
$$




$$
(0, b)=(-a, 0)+(a, b) .
$$

So in this case (i) and (ii) are equivalent.

Example 5. According to (iii) of Theorem 1, the ideal $I(4,6)$ of $2 \mathbf{N} \times 2 \mathbf{N}$ is not directly decomposable since

$$
\begin{aligned}
(4,0),(0,6) \notin I(4,6) & =(4,6) \mathbb{N}+(4,6)(2 \mathbb{N} \times 2 \mathbb{N})=(4,6) \mathbb{N}+(8 \mathbb{N} \times 12 \mathbb{N})= \\
& =(8 \mathbb{N} \times 12 \mathbb{N}) \cup((4+8 \mathbb{N}) \times(6+12 \mathbb{N})) .
\end{aligned}
$$

Next we present several simple sufficient conditions for direct decomposability of ideals.

Corollary 1. Let $\mathbf{S}_{1}$ and $\mathbf{S}_{2}$ be commutative semirings such that one of the following conditions hold:

(i) $\mathbf{S}_{1}$ and $\mathbf{S}_{2}$ are unitary,

(ii) $\mathbf{S}_{1}$ is unitary and $\mathbf{S}_{2}$ is idempotent,

(iii) $\mathbf{S}_{1}$ and $\mathbf{S}_{2}$ are idempotent,

(iv) $\mathbf{S}_{1}$ and $\mathbf{S}_{2}$ are rings and $\mathbf{I d}\left(\mathbf{S}_{1} \times \mathbf{S}_{2}\right)$ is distributive.

Then every ideal of $\mathbf{S}_{1} \times \mathbf{S}_{2}$ is directly decomposable.

Proof. Assume $(a, b) \in I \in \operatorname{Id}\left(\mathbf{S}_{1} \times \mathbf{S}_{2}\right)$. Then

$(a, 0)=(a, b)(1,0) \in I$ and $(0, b)=(a, b)(0,1) \in I$ in case (i),

$(a, 0)=(a, b)(1,0) \in I$ and $(0, b)=(a, b)(0, b) \in I$ in case (ii) and

$(a, 0)=(a, b)(a, 0) \in I$ and $(0, b)=(a, b)(0, b) \in I$ in case (iii)

showing direct decomposability of $I$ according to condition (iii) of Theorem 1 . In case (iv), direct decomposability of $I$ follows from condition (ii) of Theorem 1 together with Remark 1.

If a field $\mathbf{F}=(F,+, \cdot)$ is considered as a ring then it has no proper ideals. However, the same is valid also in the case of semiring ideals. Namely, if $I$ is a non-zero semiring ideal in $\mathbf{F}$ and $d \in I \backslash\{0\}$, then for each $x \in F$ we have $x=d d^{-1} x \in I$ proving $I=F$.

Proposition 1. If $\mathbf{S}$ is a commutative semiring and $\mathbf{F}$ a field then every ideal of $\mathbf{S} \times \mathbf{F}$ is directly decomposable.

Proof. Assume $\mathbf{S}=(S,+, \cdot, 0)$ and $\mathbf{F}=(F,+, \cdot, 0)$, let $I \in \operatorname{Id}(\mathbf{S} \times \mathbf{F})$ and put $J:=$ $\pi_{1}(I)$. If $I \subseteq S \times\{0\}$ then $I=J \times\{0\}$. Now assume $I \nsubseteq S \times\{0\}$. Then there exists some $(a, b) \in I$ with $b \neq 0$. If $(c, d) \in J \times F$ then $c \in J=\pi_{1}(I)$. Thus there exists some $e \in F$ with $(c, e) \in I$ and hence

$$
(c, d)=(c, e)+(a, b)\left(0, b^{-1}(d-e)\right) \in I
$$

showing $I=J \times F$. Hence, $\mathbf{S} \times \mathbf{F}$ has directly decomposable ideals. 


\section{CONGRUENCE KERNELS OF DIRECT PRODUCTS OF COMMUTATIVE SEMIRINGS}

Now we draw our attention to congruence kernels.

If $\Theta_{1} \in \operatorname{Con} \mathbf{S}_{1}$ and $\Theta_{2} \in \mathbf{S}_{2}$ then

$$
\Theta_{1} \times \Theta_{2}:=\left\{\left(\left(x_{1}, x_{2}\right),\left(y_{1}, y_{2}\right)\right) \mid\left(x_{1}, y_{1}\right) \in \Theta_{1},\left(x_{2}, y_{2}\right) \in \Theta_{2}\right\} \in \operatorname{Con}\left(\mathbf{S}_{1} \times \mathbf{S}_{2}\right)
$$

and $[(0,0)]\left(\Theta_{1} \times \Theta_{2}\right)=[0] \Theta_{1} \times[0] \Theta_{2}$. However, there may exist congruences $\Theta$ on $\mathbf{S}_{1} \times \mathbf{S}_{2}$ such that $[(0,0)] \Theta \neq[0] \Theta_{1} \times[0] \Theta_{2}$ for all possible $\Theta_{1} \in \operatorname{Con} \mathbf{S}_{1}$ and $\Theta_{2} \in$ $\operatorname{Con} \mathbf{S}_{2}$. It should be noted that Fraser and Horn (cf. [4]) presented necessary and sufficient conditions for direct decomposability of congruences. In the following we will modify these conditions for congruence kernels.

If $\mathbf{S}_{1}=\left(S_{1},+, \cdot, 0\right)$ and $\mathbf{S}_{2}=\left(S_{2},+, \cdot, 0\right)$ are commutative semirings, $\Theta \in \operatorname{Con}\left(\mathbf{S}_{1} \times\right.$ $\left.\mathbf{S}_{2}\right)$ and $i \in\{1,2\}$ then we put

$$
\begin{aligned}
\pi_{i}(\Theta) & :=\left\{\left(\pi_{i}(x), \pi_{i}(y)\right) \mid(x, y) \in \Theta\right\}, \\
\Pi_{i} & :=\left\{(x, y) \in\left(S_{1} \times S_{2}\right)^{2} \mid \pi_{i}(x)=\pi_{i}(y)\right\} .
\end{aligned}
$$

Note that $\Theta_{i}:=\pi_{i}(\Theta) \in \operatorname{Con}\left(\mathbf{S}_{i}\right), \Pi_{i} \in \operatorname{Con}\left(\mathbf{S}_{1} \times \mathbf{S}_{2}\right)$ and $\Pi_{1} \cap \Pi_{2}=\{(x, x) \mid x \in$ $\left.S_{1} \times S_{2}\right\}$. Let us remark that in general $[0] \Theta_{i} \neq \pi_{i}([(0,0)] \Theta)$, namely e.g. $a \in[0] \Theta_{1}$ is equivalent to the fact that there exist $b, c \in S_{2}$ with $(a, b) \in[(0, c)] \Theta$.

We call the kernel $[(0,0)] \Theta$ directly decomposable if

$$
[(0,0)] \Theta=\pi_{1}([(0,0)] \Theta) \times \pi_{2}([(0,0)] \Theta),
$$

and furthermore, we call the kernel $[(0,0)] \Theta$ strongly directly decomposable if

$$
[(0,0)] \Theta=[0] \Theta_{1} \times[0] \Theta_{2} .
$$

Note that

$$
\begin{aligned}
\pi_{i}([(0,0)] \Theta) & \subseteq[0] \Theta_{i} \text { for } i=1,2, \\
{[(0,0)] \Theta } & \subseteq \pi_{1}([(0,0)] \Theta) \times \pi_{2}([(0,0)] \Theta),
\end{aligned}
$$

thus strongly direct decomposability implies direct decomposability (cf. also the following Theorems 2 and 4).

We characterize strongly directly decomposable congruence kernels as follows:

Theorem 2. If $\mathbf{S}_{1}=\left(S_{1},+, \cdot, 0\right)$ and $\mathbf{S}_{2}=\left(S_{2},+, \cdot, 0\right)$ are commutative semirings and $\Theta \in \operatorname{Con}\left(\mathbf{S}_{1} \times \mathbf{S}_{2}\right)$ then $[(0,0)] \Theta$ is strongly directly decomposable if and only if the following holds:

$$
\text { If }(a, b) \Theta(0, c) \text { and }(d, e) \Theta(f, 0) \text { then }(a, e) \Theta(0,0)
$$

for $(a, b),(0, c),(d, e),(f, 0) \in S_{1} \times S_{2}$.

Proof. If $[(0,0)] \Theta$ is strongly directly decomposable and

$$
(a, b) \Theta(0, c) \text { and }(d, e) \Theta(f, 0)
$$


for $(a, b),(0, c),(d, e),(f, 0) \in S_{1} \times S_{2}$ then $(a, e) \in[0] \Theta_{1} \times[0] \Theta_{2}=[(0,0)] \Theta$. If, conversely, the condition of the theorem holds and $(g, h) \in[0] \Theta_{1} \times[0] \Theta_{2}$ then there exist $i, j \in S_{1}$ and $k, l \in S_{2}$ with $(g, k) \Theta(0, l)$ and $(i, h) \Theta(j, 0)$ and hence $(g, h) \in$ $[(0,0)] \Theta$ showing $[0] \Theta_{1} \times[0] \Theta_{2} \subseteq[(0,0)] \Theta$. The converse inclusion is trivial.

Using this result we can prove the following

Theorem 3. If $\mathbf{S}_{1}$ and $\mathbf{S}_{2}$ are commutative semirings, $\Theta \in \operatorname{Con}\left(\mathbf{S}_{1} \times \mathbf{S}_{2}\right)$ and

$$
\begin{aligned}
& {[(0,0)]\left(\left(\Theta \vee \Pi_{1}\right) \cap \Pi_{2}\right) \subseteq[(0,0)] \Theta,} \\
& {[(0,0)]\left(\left(\Theta \vee \Pi_{2}\right) \cap \Pi_{1}\right) \subseteq[(0,0)] \Theta}
\end{aligned}
$$

then $[(0,0)] \Theta$ is strongly directly decomposable.

Proof. Let $(a, b),(0, c),(d, e),(f, 0) \in S_{1} \times S_{2}$ and assume $(a, b) \Theta(0, c)$ and $(d, e) \Theta(f, 0)$. Then

$$
(a, 0) \Pi_{1}(a, b) \Theta(0, c) \Pi_{1}(0,0) \text { and }(a, 0) \Pi_{2}(0,0)
$$

and hence

$$
(a, 0) \in[(0,0)]\left(\left(\Theta \vee \Pi_{1}\right) \cap \Pi_{2}\right) \subseteq[(0,0)] \Theta .
$$

Analogously,

$$
(0, e) \Pi_{2}(d, e) \Theta(f, 0) \Pi_{2}(0,0) \text { and }(0, e) \Pi_{1}(0,0)
$$

and hence

$$
(0, e) \in[(0,0)]\left(\left(\Theta \vee \Pi_{2}\right) \cap \Pi_{1}\right) \subseteq[(0,0)] \Theta .
$$

Therefore

$$
((a, 0)+(0, e)) \Theta((0,0)+(0,0)) .
$$

Since

$$
\begin{aligned}
& (a, e)=(a, 0)+(0, e), \\
& (0,0)=(0,0)+(0,0),
\end{aligned}
$$

we obtain

$$
(a, e) \in[(0,0)] \Theta .
$$

Now the assertion follows from Theorem 2.

Recall that an algebra $\mathbf{A}$ with 0 is called distributive at 0 (see e.g. [1]) if for all $\Theta, \Phi, \Psi \in$ Con $\mathbf{A}$ we have

$$
[0]((\Theta \vee \Phi) \cap \Psi)=[0]((\Theta \cap \Psi) \vee(\Phi \cap \Psi)) .
$$

A class of algebras with 0 is called distributive at 0 if any of its members has this property. Applying Theorem 3 we can state:

Corollary 2. If $\mathbf{S}_{1}$ and $\mathbf{S}_{2}$ are commutative semirings and $\mathbf{S}_{1} \times \mathbf{S}_{2}$ is distributive at $(0,0)$ then the kernel of every congruence on $\mathbf{S}_{1} \times \mathbf{S}_{2}$ is strongly directly decomposable. 
Proof. For all $\Theta \in \operatorname{Con}\left(\mathbf{S}_{1} \times \mathbf{S}_{2}\right)$ we have

$$
\begin{aligned}
{[(0,0)]\left(\left(\Theta \vee \Pi_{1}\right) \cap \Pi_{2}\right) } & =[(0,0)]\left(\left(\Theta \cap \Pi_{2}\right) \vee\left(\Pi_{1} \cap \Pi_{2}\right)\right)=[(0,0)]\left(\Theta \cap \Pi_{2}\right) \subseteq \\
& \subseteq[(0,0)] \Theta, \\
{[(0,0)]\left(\left(\Theta \vee \Pi_{2}\right) \cap \Pi_{1}\right) } & =[(0,0)]\left(\left(\Theta \cap \Pi_{1}\right) \vee\left(\Pi_{2} \cap \Pi_{1}\right)\right)=[(0,0)]\left(\Theta \cap \Pi_{1}\right) \subseteq \\
& \subseteq[(0,0)] \Theta .
\end{aligned}
$$

Now the result follows from Theorem 3.

Recall the Mal'cev type characterization of distributivity at 0 from [1] (Theorem 8.2.2):

Proposition 2. A variety with 0 is distributive at 0 if and only if there exist some positive integer $n$ and binary terms $t_{0}, \ldots, t_{n}$ such that

$$
\begin{aligned}
& t_{0}(x, y) \approx 0, \\
& t_{i}(0, y) \approx 0 \text { for } i \in\{0, \ldots, n\}, \\
& t_{i}(x, 0) \approx t_{i+1}(x, 0) \text { for even } i \in\{0, \ldots, n-1\}, \\
& t_{i}(x, x) \approx t_{i+1}(x, x) \text { for odd } i \in\{0, \ldots, n-1\}, \\
& t_{n}(x, y) \approx x .
\end{aligned}
$$

We can apply Proposition 2 to idempotent commutative semirings.

Corollary 3. The variety of idempotent commutative semirings is distributive at 0 and hence has strongly directly decomposable congruence kernels.

Proof. If

$$
\begin{aligned}
n & :=2, \\
t_{0}(x, y) & :=0, \\
t_{1}(x, y) & :=x y, \\
t_{2}(x, y) & :=x
\end{aligned}
$$

then

$$
\begin{aligned}
& t_{0}(x, y) \approx 0, \\
& t_{0}(0, y) \approx 0, \\
& t_{1}(0, y) \approx 0 y \approx 0, \\
& t_{2}(0, y) \approx 0, \\
& t_{0}(x, 0) \approx 0 \approx x 0 \approx t_{1}(x, 0), \\
& t_{1}(x, x) \approx x x \approx x \approx t_{2}(x, x), \\
& t_{2}(x, y) \approx x
\end{aligned}
$$

and hence, by Proposition 2, we obtain the result. 
The following characterization of directly decomposable congruence kernels is similar to the characterization presented in Theorem 2.

Theorem 4. For commutative semirings $\mathbf{S}_{1}=\left(S_{1},+, \cdot, 0\right)$ and $\mathbf{S}_{2}=\left(S_{2},+, \cdot, 0\right)$ and $\Theta \in \operatorname{Con}\left(\mathbf{S}_{1} \times \mathbf{S}_{2}\right)$ the kernel $[(0,0)] \Theta$ is directly decomposable if and only if

$$
(a, b),(c, d) \in[(0,0)] \Theta \text { implies }(a, d) \in[(0,0)] \Theta .
$$

Proof. If the kernel $[(0,0)] \Theta$ is directly decomposable and $(a, b),(c, d) \in$ $\in[(0,0)] \Theta$ then

$$
a \in \pi_{1}([(0,0)] \Theta) \text { and } d \in \pi_{2}([(0,0)] \Theta)
$$

whence

$$
(a, d) \in \pi_{1}([(0,0)] \Theta) \times \pi_{2}([(0,0)] \Theta)=[(0,0)] \Theta
$$

proving (3.1). Conversely, assume (3.1) to be satisfied and let

$$
(a, d) \in \pi_{1}([(0,0)] \Theta) \times \pi_{2}([(0,0)] \Theta) .
$$

Then there exist $b \in S_{2}$ and $c \in S_{1}$ with $(a, b),(c, d) \in[(0,0)] \Theta$. Using (3.1) we conclude $(a, d) \in[(0,0)] \Theta$ proving

$$
\pi_{1}([(0,0)] \Theta) \times \pi_{2}([(0,0)] \Theta) \subseteq[(0,0)] \Theta .
$$

The converse inclusion is trivial.

It is evident also from the conditions of Theorems 2 and 4 that if a direct product of semirings has strongly directly decomposable congruence kernels then it has directly decomposable congruence kernels.

We say that a class $C$ of algebras of the same type containing a constant 0 has $\mathrm{dir}$ ectly decomposable congruence kernels if for any $\mathbf{A}_{1}, \mathbf{A}_{2} \in \mathcal{C}$ and each $\Theta \in \operatorname{Con}\left(\mathbf{A}_{1} \times\right.$ $\left.\mathbf{A}_{2}\right),[(0,0)] \Theta$ is directly decomposable.

The following Mal'cev type condition was derived in [1]:

Proposition 3 (Theorem 11.0.4 in [1]). A variety of algebras with 0 has directly decomposable congruence kernels if there exist positive integers $m$ and $n$, binary terms $s_{1}, \ldots, s_{m}, t_{1}, \ldots \ldots, t_{m}$ and $(m+2)$-ary terms $u_{1}, \ldots, u_{n}$ satisfying the identities

$$
\begin{aligned}
u_{1}\left(x, y, s_{1}(x, y), \ldots, s_{m}(x, y)\right) & \approx x, \\
u_{1}\left(y, x, t_{1}(x, y), \ldots, t_{m}(x, y)\right) & \approx x, \\
u_{i}\left(y, x, s_{1}(x, y), \ldots, s_{m}(x, y)\right) & \approx u_{i+1}\left(x, y, s_{1}(x, y), \ldots, s_{m}(x, y)\right) \text { for } i= \\
& =1, \ldots, n-1, \\
u_{i}\left(x, y, t_{1}(x, y), \ldots, t_{m}(x, y)\right) & \approx u_{i+1}\left(y, x, t_{1}(x, y), \ldots, t_{m}(x, y)\right) \text { for } i= \\
& =1, \ldots, n-1, \\
u_{n}\left(y, x, s_{1}(x, y), \ldots, s_{m}(x, y)\right) & \approx x, \\
u_{n}\left(x, y, t_{1}(x, y), \ldots, t_{m}(x, y)\right) & \approx y .
\end{aligned}
$$


Corollary 4. The variety of unitary commutative semirings has directly decomposable congruence kernels.

Proof. If

$$
\begin{aligned}
m & :=3, \\
n & :=2, \\
s_{1}(x, y) & :=1, \\
s_{2}(x, y) & :=0, \\
s_{3}(x, y) & :=0, \\
t_{1}(x, y) & :=0, \\
t_{2}(x, y) & :=1, \\
t_{3}(x, y) & :=y, \\
u_{1}(x, y, z, u, v) & :=x z+y u, \\
u_{2}(x, y, z, u, v) & :=y z+v
\end{aligned}
$$

then

$$
\begin{aligned}
& u_{1}(x, y, 1,0,0) \approx x 1+y 0 \approx x, \\
& u_{1}(y, x, 0,1, y) \approx y 0+x 1 \approx x, \\
& u_{1}(y, x, 1,0,0) \approx y 1+x 0 \approx y \approx y 1+0 \approx u_{2}(x, y, 1,0,0), \\
& u_{1}(x, y, 0,1, y) \approx x 0+y 1 \approx y \approx x 0+y \approx u_{2}(y, x, 0,1, y), \\
& u_{2}(y, x, 1,0,0) \approx x 1+0 \approx x, \\
& u_{2}(x, y, 0,1, y) \approx y 0+y \approx y
\end{aligned}
$$

and hence, by Proposition 3, we obtain the result.

\section{Conclusion}

Although not all ideals of a commutative semiring are congruence kernels, we obtained a characterization of direct decomposability of ideals on a direct product of commutative semirings analogous to that by Fraser-Horn for congruences. Using a Mal'cev type condition characterizing varieties which are distributive at 0 , we have shown that the variety of idempotent commutative semirings has strongly directly decomposable congruence kernels. Moreover, we proved that the variety of unitary commutative semirings has directly decomposable congruence kernels. This generalizes the corresponding result for the variety of unitary commutative rings which is well known.

\section{ACKNOWLEDGEMENT}

The authors thank the anonymous referee for his/her valuable suggestions. 


\section{REFERENCES}

[1] I. Chajda, G. Eigenthaler, and H. Länger, Congruence classes in universal algebra. Lemgo: Heldermann Verlag, 2003.

[2] I. Chajda, G. Eigenthaler, and H. Länger, "Ideals of direct products of rings." Asian-Eur. J. Math., vol. 11, p. 1850094 (6 pages), 2018.

[3] I. Chajda and H. Länger, "Ideals and their complements in commutative semirings." Soft Computing, vol. 23, pp. 5385-5392, 2019.

[4] G. A. Fraser and A. Horn, "Congruence relations in direct products." Proc. Am. Math. Soc., vol. 26, pp. 390-394, 1970.

[5] J. S. Golan, Semirings and their applications. Dordrecht: Kluwer Academic Publishers, 1999.

[6] W. Kuich and A. Salomaa, Semirings, automata, languages. Springer, Berlin, 1986, vol. 5.

Authors' addresses

Ivan Chajda

Palacký University Olomouc, Department of Algebra and Geometry, 17. listopadu 12, 77146 Olomouc, Czech Republic

E-mail address: ivan.chajda@upol.cz

\section{Günther Eigenthaler}

TU Wien, Institute of Discrete Mathematics and Geometry, Wiedner Hauptstraße 8-10, 1040 Vienna, Austria

E-mail address: guenther.eigenthaler@tuwien.ac.at

Helmut Länger

TU Wien, Institute of Discrete Mathematics and Geometry, Wiedner Hauptstraße 8-10, 1040 Vienna, Austria, and Palacký University Olomouc, Department of Algebra and Geometry, 17. listopadu 12, 771 46 Olomouc, Czech Republic

E-mail address: helmut. laengeretuwien.ac.at 